Mathematical Research Letters 6, 779-793 (1999)

\title{
TAYLOR AND MINIMAL RESOLUTIONS OF HOMOGENEOUS POLYNOMIAL IDEALS
}

\author{
SERGEy YuZvinsky
}

\section{Introduction}

In the theory of monomial ideals of a polynomial ring $S$ over a field $k$, it is convenient that for each such ideal $I$ there is a standard free resolution, a so called Taylor resolution, that can be canonically constructed from the minimal system of monomial generators of $I$ (see [7], pp. 439 and section 2). On the other hand no construction of a minimal resolution for an arbitrary monomial ideal has been known. Recently a minimal resolution was constructed in [4] for a class of so called generic monomial ideals. Also in $[2,10,11]$ various invariants of monomial ideals were related to combinatorics of the lattice $D$ of the least common multiples $(\mathrm{lcm})$ of generating monomials. In particular, in [11] the Betti numbers of the $S$-module $S / I$ were expressed through homology of $D$ and it was proved that even the algebra structure of $\operatorname{Tor}_{*}^{S}(S / I, k)$ was defined by that lattice although an explicit formula was not given in that paper.

Given a system of generators of an arbitrary ideal $I$ of $S$, one can factor the generators in products of irreducibles and construct the Taylor complex similarly to the Taylor resolution of a monomial ideals. In general this complex is not acyclic. One non-monomial case where it is acyclic has been used in [15].

In the present paper, a necessary and sufficient condition is given for the Taylor complex of a system $\mathcal{A}$ of homogeneous polynomials to be acyclic (Theorem 2.4). This condition involves the local homology of the lattice $D$ of lcm of elements from $\mathcal{A}$ and the depth of ideals generated by their irreducible factors. If this condition holds then the Betti numbers of $S / I$ are defined by the local homology of $D$ similarly to the case of monomial ideals (Theorem 2.5). Moreover in section 3 we exhibit a DGA defined by combinatorics whose cohomology algebra is isomorphic to the algebra $\operatorname{Tor}_{*}^{S}(S / I, k)$. This construction makes sense for arbitrary extrinsically graded lattices (see definition in section 3 ) and generalizes the DGA constructed in $[16,17]$ for a cohomology algebra of a complex subspace complement. Section 4 contains what can be considered the main result of the paper (Theorem 4.3). There, for any ideal $I$ having a Taylor resolution we give a combinatorial construction of a subcomplex of it that is a minimal resolution of $S / I$. This construction is not canonical and involves computations of homology of posets which hardly can be avoided in general. We also describe completely

Received May 27, 1999. 
the class of ideals for which our minimal resolution reduces to the minimal resolution from [4]. Finally in section 5 we give examples of classes of $\mathcal{A}$ satisfying the condition of Theorem 2.4. For instance we consider the ideals whose generators are products of linear polynomials which is important for theory of hyperplane and subspace arrangements.

The author is grateful to D. Eisenbud for discussions of the results of the paper. In particular, the proof of Theorem 4.3 would have been longer without his advice.

\section{Taylor complex}

Let $S=k\left[x_{1}, \ldots, x_{n}\right]$ be the polynomial ring over a field $k, \mathcal{A}=\left\{Q_{1}, \ldots, Q_{m}\right\}$ a set of homogeneous polynomials from $S$, and $I=I(\mathcal{A})$ the ideal of $S$ generated by $\mathcal{A}$. In this section we give a necessary and sufficient condition for the Taylor complex of $\mathcal{A}$ to be a resolution of the $S$-module $S / I$. We can assume that $\mathcal{A}$ generates $I$ minimally, in particular, none of polynomials from $\mathcal{A}$ divides another one. Also, we will always regard all the subsets of $\mathcal{A}$ provided with the ordering induces by a fixed linear ordering on $\mathcal{A}$.

Let $K: 0 \rightarrow K_{m} \rightarrow \cdots \rightarrow K_{1} \rightarrow K_{0}=k \rightarrow 0$ be the augmented shifted by -1 chain complex over $k$ of the simplex whose set of vertices is $\mathcal{A}$. Explicitly the linear space $K_{p}$ has a basis consisting of all the subsets of $\mathcal{A}$ with $p$ elements. The differential $d_{p}: K_{p} \rightarrow K_{p-1}$ is given with respect to these bases by the matrix with entries $d_{\sigma, \tau}(\sigma, \tau \in \mathcal{A},|\sigma|=p-1,|\tau|=p)$ equal to $(-1)^{\epsilon(\tau, i)}$ if $\sigma=\tau \backslash\left\{Q_{i}\right\}$ where $\epsilon(\tau, i)=\left|\left\{Q_{j} \in \tau \mid j<i\right\}\right|$ and 0 if $\sigma \not \subset \tau$. For each $\sigma \subset \mathcal{A}$ denote by $Q_{\sigma}$ the lcm of all $Q_{i} \in \sigma$.

Definition 2.1. The Taylor complex of $\mathcal{A}$ is the complex $\tilde{K}$ of the free $S$ modules $\tilde{K}_{p}=K_{p} \otimes S$ with the differentials $\tilde{d}_{p}: \tilde{K}_{p} \rightarrow \tilde{K}_{p-1}$ given by the matrix $\tilde{d}_{\sigma, \tau}=\left(Q_{\tau} / Q_{\sigma}\right) d_{\sigma, \tau}$.

Notice that $H_{0}(\tilde{K})=S / I$. Our goal is to give equivalent conditions for the acyclisity of $\tilde{K}$.

We will be dealing with finite posets and need to introduce some notation. To every poset one can assign its complex of flags (i.e., increasing sequences of elements) and attribute the topological invariants of this complex to the poset itself. In this sense we will use homology of a poset and homotopy equivalence of two of them. If $L$ is a lattice with the minimal element $\hat{0}$ and the maximal one $\hat{1}$ then the flag complex of $L \backslash\{\hat{0}, \hat{1}\}$ is homotopy equivalent to its atomic and coatomic complexes (e.g., see [5]). For instance, recall that the atomic complex is the abstract complex whose vertices are all the atoms of $L$ and a set of atoms is a simplex if it is bounded above in $L \backslash\{\hat{1}\}$. If $\sigma \subset L$ then $\bigvee(\sigma)$ denotes the least upper bound (join) of $\sigma$. For every $X \in L$ we put $L_{\leq X}=(\hat{0}, X]=\{Y \in L \mid 0<Y \leq X\}$ and $L_{<X}=L_{\leq X} \backslash\{X\}$.

Now denote by $D=D(\mathcal{A})$ the lattice of the lcm of all subsets of $\mathcal{A}$ ordered by divisibility. This lattice is provided with the monotone map $\phi: \mathcal{B} \rightarrow D$ from the Boolean lattice $\mathcal{B}$ of all the subsets of $\mathcal{A}$ given by $\phi(\sigma)=Q_{\sigma}$. Denote by $D_{0}$ 
the poset $D$ with the smallest element (constant polynomial 1) deleted and call a subset of a poset decreasing if with each element it contains all smaller ones.

Lemma 2.2. For every decreasing subset $F$ of $D_{0}$ the restriction of $\phi$ to $\phi^{-1}(F)$ is a homotopy equivalence.

Proof. For $Q \in F$ put $\mathcal{B}(\leq Q)=\phi^{-1}(\{P \in F \mid P \leq Q\})$ and notice that the poset $\mathcal{B}(\leq Q)$ has the unique maximal element equals $\left\{Q_{i} \in \mathcal{A} \mid Q_{i}\right.$ divides $\left.Q\right\}$. Thus $\mathcal{B}(Q)$ is contractable and the restriction of $\phi$ is a homotopy equivalence (cf. [14]).

The lattice $D$ is naturally embedded as a join-sublattice in a larger polynomial lattice $W$. Let $E$ be the set of all irreducible factors of elements from $\mathcal{A}$ (chosen one from each equivalence class). Notice that $E$ depends on the fixed field $k$. Let $W$ be the lattice of all the products of elements from $E$ ordered by divisibility (repetitions are allowed). Clearly $W$ is isomorphic as a partially ordered semigroup to $\mathbb{N}^{k}(k=|E|)$.

To study the complex $\tilde{K}$ we use the evaluation at vectors $v \in \bar{k}^{n}$ where $\bar{k}$ is the algebraic closure of $k$. This means that for every $v \in \bar{k}^{n}$ we consider the complex $K(v)$ with $K(v)_{p}=K_{p} \otimes \bar{k}$ and $d(v)_{p}: K(v)_{p+1} \rightarrow K(v)_{p}$ given by the matrix $d(v)_{\sigma, \tau}=\tilde{d}_{\sigma, \tau}(v)$. On the other hand, every $v$ defines a subset $E_{v}$ of $E$ of all the elements of $E$ that vanish at $v$. Denote by $W(v)$ the sublattice of $W$ of all the elements of $W$ whose all irreducible factors are from $E_{v}$ and by $\psi_{v}$ the monotone map ("projection") $D \rightarrow W(v)$ defined by

$$
\psi_{v}(Q)=\max \{P \in W(v) \mid P \leq Q\} .
$$

For each $P \in W(v)$ we put $D(v,<P)=\left\{Q \in D \mid \phi_{v}(Q)<P\right\}$. Besides every $P \in W(v)$ defines the subcomplex $K_{v, P}$ of $K \otimes \bar{k}$ spanned by $\sigma \subset \mathcal{A}$ such that $\psi_{v}\left(Q_{\sigma}\right)=P$. Here and in the rest of the paper we consider only $P \in W(v)$ such that $P \in \psi_{v}(D)$. Substituting in the last definition "=" by " $\leq$ " or " $<$ " we obtain complexes $K_{v, \leq P}$ and $K_{v,<P}$ respectively. Clearly $K_{v, P}=K_{v, \leq P} / K_{v,<P}$.

Notice that if $E(v)=\emptyset$ then $W(v)=\{1\}$ and $K(v) \simeq K$. We need the following lemma.

\section{Lemma 2.3.}

(i) $K(v) \simeq \oplus_{P \in \psi_{v}(D)} K_{v, P}$ for every $v \in \bar{k}^{n}$.

(ii) The complex $K_{v, \leq P}$ is acyclic for every $v$ and $P \in \psi_{v}(D)$.

(iii) The complex $K_{v,<P}$ is homotopy equivalent to the chain complex of $D(v,<$ $P)$ shifted by -1 .

Proof. (i) The decomposition of $K(v)$ in the direct sum according to $\psi_{v}\left(Q_{\sigma}\right)$ follows from definitions. The required isomorphism can be given by $\sigma \mapsto\left(Q_{\sigma} / P\right)(v) \sigma$ where $P=\psi_{v}\left(Q_{\sigma}\right)$.

(ii) The complex $K_{v, \leq P}$ is the chain complex of the simplex on the vertices $Q_{i} \in \mathcal{A}$ with $\psi_{v}\left(Q_{i}\right) \leq P$. The result follows.

(iii) The complex $K_{v,<P}$ is the shifted by -1 chain complex of an abstract complex whence it is homotopy equivalent to the poset of its non-empty simplexes 
ordered by inclusion. More explicitly $K_{v,<P}$ is homotopy equivalent (after the shift) to the subposet $\phi^{-1}(D(v,<P))$ of $\mathcal{B}$. Since $D(v,<P)$ is decreasing we can apply Lemma 2.2 which completes the proof.

It will be convenient for us to express properties of $v$ in terms of the set $E_{v}$ of irreducible polynomials. Notice that these sets can be characterized intrinsically as subsets $G \subset E$ such that the ideal $J(G)$ generated by $G$ does not contain a power of an element from $E \backslash G$. We will call those sets saturated. For every two $v, v^{\prime}$ such that $E_{v}=E_{v^{\prime}}$ we have $\psi_{v}=\psi_{v^{\prime}}$. Thus we will often use $\psi_{G}, W(G)$, and $D(G,<P)$ for $P \in W(G)$ instead of $\psi_{v}, W(v)$, and $D(v,<P)$ respectively for $v$ such that $E_{v}=G$.

Theorem 2.4. The Taylor complex of $\mathcal{A}$ is a resolution of $S / I(\mathcal{A})$ if and only if for every saturated set $G \subset E$ and $P \in \psi_{G}(D)$ we have $\tilde{H}_{p}(D(G,<P))=0$ for every $p$ such that $p \geq \operatorname{depth}(J(G))-1$.

Proof. According to the celebrated Buchsbaum-Eisenbud criterion (see for example [12], Sect. 6.4, Theorem 15) the complex $\tilde{K}$ is a resolution of $S / I$ if and only if the following three conditions hold:

(a) $\operatorname{depth} F\left(\tilde{d}_{p}\right) \geq p$ for every $p=1,2, \ldots$ where $F\left(\tilde{d}_{p}\right)$ (or shortly $F_{p}$ ) is the Fitting ideal of $\tilde{d}_{p}$;

(b) $\operatorname{rk} \tilde{d}_{m}=\operatorname{rk} \tilde{K}_{m}$;

(c) $\operatorname{rk} \tilde{d}_{p+1}+\operatorname{rk} \tilde{d}_{p}=\operatorname{rk} \tilde{K}_{p}$ for $1 \leq p<m$.

Consider $K(v)$ for a $v \in \bar{k}^{n}$. Clearly we have $\operatorname{rk} \tilde{K}_{p}=\operatorname{dim} K_{p}$ and $\operatorname{rk} d_{p}(v) \leq$ $\operatorname{rk}_{S} \tilde{d}_{p}$ for all $p$. Using these and choosing $v$ so that $E_{v}=\emptyset$ we can obtain by induction on $p$ that $\operatorname{rk}_{S} \tilde{d}_{p}=\operatorname{rk} d_{p}$ and the conditions (b) and (c) (cf. [15], Theorem 1.3). Thus we will focus on the condition (a).

For an arbitrary $v$, Lemma 2.3 (i) and (ii) and the exact sequence of the pair $\left(K_{v, \leq P}, K_{v,<P}\right)\left(P \in \psi_{v}(D)\right)$ give for all $p$

$$
H_{p}(K(v))=\oplus_{P \in \psi_{v}(D)} H_{p-1}\left(K_{v,<P}\right) .
$$

Then Lemma 2.3 (iii) gives the following equivalent form of (2.1)

$$
H_{p}(K(v))=\oplus_{P \in \psi_{v}(D)} \tilde{H}_{p-2}(D(v,<P)) .
$$

Now we include the Fitting ideals into consideration. Clearly the statement $H_{p}(K(v)) \neq 0$ is equivalent to $\operatorname{rk} d_{i}(v)<\operatorname{rk} d_{i}$ for $i=p$ or $i=p+1$ which is equivalent to the inclusion $v \in \mathcal{V}\left(F_{p+1}\right) \cup \mathcal{V}\left(F_{p}\right)$. Here and in the rest of the paper for any ideal $J$ of $S$ we denote by $\mathcal{V}(J)$ its variety in $\bar{k}^{n}$. In particular the left hand side of (2.2) is nonzero for $v$ from a closed algebraic subset of $\bar{k}^{n}$. The right hand side of $(2.2)$ is nonzero for $v$ if there exists $P \in \psi_{v}(D)$ (whence $v \in \mathcal{V}(J(P)))$ such that

$$
\tilde{H}_{p-2}(D(v,<P)) \neq 0 .
$$

If the condition (2.3) holds for one $v_{0} \in J(\mathcal{V}(P))$ it may happen that it holds only for $v$ from a closed algebraic subset of smaller dimension. On the other 
hand if we put $G=E_{v_{0}}$ then (2.3) holds for the same $P$ and $v$ from the open dense subset of $\mathcal{V}\left(J(G)\right.$ ) (of all $v$ such that $E_{v}=G$ ). Thus (2.2) implies

$$
\mathcal{V}\left(F_{p+1}\right) \cup \mathcal{V}\left(F_{p}\right)=\bigcup_{G \in \mathcal{S}_{p}} \mathcal{V}(J(G))
$$

where $\mathcal{S}_{p}$ consists of all saturated subsets $G$ of $E$ such that for each of them there exists $P \in \psi_{G}(D)$ satisfying (2.3) for some (whence every) $v$ with $E_{v}=G$. Applying the Nullstellensatz we can rewrite (2.4) in the form

$$
\bar{F}_{p+1} \cap \bar{F}_{p}=\bigcap_{G \in \mathcal{S}(p)} \bar{J}(G)
$$

where the bar means the radical of an ideal.

Now we are ready to prove that the condition (a) above is equivalent to the condition of the theorem. We are going to use that for any proper ideal there exists containing it prime ideal of the same depth (see for example [12], 5.5, Theorem 16).

Suppose that depth $F_{p} \geq p$ for every $p$. Then due to (2.5), the same is true for any prime ideal containing $\bar{J}(G)$ for $G \in \mathcal{S}(p)$ whence for any ideal $J(G)$. In other words if for some $G \subset E$ as in the condition of the theorem $r \geq \operatorname{depth}(J(G))-1$ then $G \notin \mathcal{S}(r+2)$, i.e., for every $v$ with $E_{v}=G$ and $P \in \psi_{G}(D)$ we have $\tilde{H}_{r}(D(v,<P))=0$. This is precisely the condition of the theorem.

Conversely suppose that the condition of the theorem holds and consider $G \in$ $\mathcal{S}(p)$ for some $p$. Then the assumption implies that depth $J(G) \geq p$ whence the same is true for any prime ideal containing either side of (2.5). This immediately implies condition (a) for $p$.

If $E$ is a subset of the set of indeterminates then Theorem 2.4 recovers the exactness of the classical Taylor resolution (cf. Corollary 5.2).

If a set $\mathcal{A}$ of homogeneous polynomials satisfies the condition of Theorem 2.4 then it is easy to give a combinatorial interpretation of the Betti numbers of $S / I(\mathcal{A})$, i.e., $b_{p}=\operatorname{dim} \operatorname{Tor}_{p}^{S}(S / I(\mathcal{A}), k)$.

Theorem 2.5. Suppose for a set $\mathcal{A}$ of homogeneous polynomials from $S$ the Taylor complex is exact. Then

$$
b_{p}=\sum_{Q \in D} \operatorname{dim} \tilde{H}_{p-2}\left(D_{<Q}\right)
$$

Proof. The complex whose homology is $\operatorname{Tor}_{*}^{S}(S / I, k)$, is $\tilde{K} \otimes_{S} k$ that coincides with $K(0)$. Clearly $W(0)=W$ whence $\psi_{0}$ is the embedding $D \subset W$. Thus $D(0,<P)=D_{<P}$ for any $P \in D=\psi_{0}(D)$. Applying Lemma 2.3 for $v=0$ we obtain the result. 
Remark 2.6. Using Lemma 2.2 and the exact sequence of a pair we can rewrite the formula for the Betti numbers as

$$
b_{p}=\sum_{Q \in D} \operatorname{dim} H_{p-1}(K(Q))
$$

where the complex $K(Q)$ is generated by $\mathcal{B}(Q)=\left\{\sigma \in \mathcal{B} \mid Q_{\sigma}=Q\right\}$ and its differential is the restriction of $d$. This should be compared with Theorem 4.3.

\section{Multiplication}

First in this section we consider a pure combinatorial set up of an extrinsically graded lattice (see definition below) and define a DGA on the relative atomic complex of this lattice. A special case of this definition was used in [16] in order to describe the rational cohomology ring of a subspace complement. In certain special cases it is known to give even the integer cohomology $[8,6]$.

Let $L$ be a lattice with the minimal element $\hat{0}$. The atoms of $L$ are provided with an arbitrary but fixed linear ordering. An extrinsic grading of $L$ is a strictly monotone map rk $L \rightarrow \mathbb{N}^{s}$ (for some positive integer $s$ ) with $\operatorname{rk}(\hat{0})=0$ and

$$
\operatorname{rk}(X \vee Y)+\operatorname{rk}(X \wedge Y) \leq \operatorname{rk}(X)+\operatorname{rk}(Y),
$$

for $X, Y \in L$. We call $L$ extrinsically graded and $\operatorname{rk} X \operatorname{rank}$ of $X$ for $X \in L$.

Let us recall the relative atomic complex $\Delta=\Delta(L)$ of $L$ (cf. [17]). It is a chain complex (over a field or $\mathbb{Z}$ ) of linear spaces (resp., free modules) whose $p$-th term $\Delta_{p}$ has a basis consisting of subsets $\sigma$ of atoms of $L$ with $|\sigma|=p$ (notice the unusual grading) and with the differential defined by

$$
d \sigma=\sum_{Z_{i} \in \sigma, V}(-1)^{\epsilon(\sigma, i)} \sigma_{i} .
$$

(Here as in section 2, $\epsilon(\omega, i)=\mid\left\{Z_{j} \in E|j<i|\right.$ and if $Z_{i} \in \omega$ then $\omega_{i}=\omega \backslash\left\{Z_{i}\right\}$ ). As usual $\Delta_{0}$ is spanned by the empty set of atoms of $L$. It is easy to see that $\Delta=\oplus_{X \in L} \Delta_{X}$ where $\Delta_{X}$ is spanned by $\sigma$ with $\bigvee(\sigma)=X$ and $\tilde{H}_{p}\left(\Delta_{X}\right)=$ $\tilde{H}_{p-2}\left(L_{<X}\right)$ where the latter natural isomorphism is given by the boundary map in an exact sequence of $\left(L_{\leq X}, L_{<X}\right)$ (with a shift of dimension).

Proposition 3.1. The complex $\Delta$ gets the structure of a DGA via the bilinear multiplication defined by

$$
\sigma \cdot \tau= \begin{cases}0 & \text { if } \operatorname{rk}(\bigvee(\sigma \cup \tau)) \neq \operatorname{rk}(\bigvee(\sigma))+\operatorname{rk}(\bigvee(\tau)), \\ (-1)^{\epsilon(\sigma, \tau)} \sigma \cup \tau & \text { otherwise }\end{cases}
$$

where $\epsilon(\sigma, \tau)$ is the parity of the permutation of $\sigma \cup \tau$ (shuffle) putting all elements of $\tau$ after elements of $\sigma$ and preserving fixed orders inside these sets.

Proof. It suffices to check the Leibniz rule. We consider two cases. 
(i) $\operatorname{rk}(\bigvee(\sigma \cup \tau)) \neq \operatorname{rk}(\bigvee(\sigma))+\operatorname{rk}(\bigvee(\tau))$. Then $\sigma \cdot \tau=0$. On the other hand, for every $\sigma_{i}$ such that $\bigvee\left(\sigma_{i}\right)=\bigvee(\sigma)$

$$
\begin{aligned}
\operatorname{rk}\left(\bigvee\left(\sigma_{i} \cup \tau\right)\right) \leq \operatorname{rk} \bigvee((\sigma \cup \tau)) & <\operatorname{rk}(\bigvee(\sigma))+\operatorname{rk}(\bigvee(\tau)) \\
& =\operatorname{rk}\left(\bigvee\left(\sigma_{i}\right)\right)+\operatorname{rk}(\bigvee(\tau)),
\end{aligned}
$$

whence $d(\sigma) \cdot \tau=0$. Similarly $\sigma \cdot d(\tau)=0$ that gives the Leibniz rule in this case.

(ii) $\operatorname{rk}(\bigvee(\sigma \cup \tau))=\operatorname{rk}(\bigvee(\sigma))+\operatorname{rk}(\bigvee(\tau))$. First notice that in this case $\sigma \cap \tau=\emptyset$ Indeed $Z \in \sigma \cap \tau$ would imply

$$
\operatorname{rk}(\bigvee(\sigma \cup \tau)) \leq \operatorname{rk}\left(\bigvee\left(\sigma_{i}\right)\right)+\operatorname{rk}(\bigvee(\tau))-\operatorname{rk} Z<\operatorname{rk}(\bigvee(\sigma))+\operatorname{rk}(\bigvee(\tau))
$$

Further, both sides of the Leibniz equality are combinations of basic elements $\omega_{i}=(\sigma \cup \tau)_{i}$. Suppose $Z_{i} \in \sigma$. Then the coefficient of $\omega_{i}$ in the left hand side is

$$
(-1)^{\epsilon(\omega, i)+\epsilon(\sigma, \tau)}
$$

and that in the right hand side side is

$$
(-1)^{\epsilon(\sigma, i)+\epsilon(\sigma, \tau)} \text {. }
$$

We have

$$
\begin{aligned}
\epsilon(\sigma, i)+\epsilon\left(\sigma_{i}, \tau\right) & \equiv \epsilon(\sigma, i)+\epsilon(\tau, i)+\epsilon(\sigma, \tau) \\
& \equiv \epsilon(\omega, i)+\epsilon(\sigma, \tau)(\bmod 2) .
\end{aligned}
$$

which implies the Leibniz equality in this case. The case where $Z_{i} \in \tau$ can be completed similarly.

Let us consider several important particular cases of this construction.

Examples. 1. If $L$ is a geometric lattice intrinsically graded by its standard rank then the complex $\Delta$ over $\mathbb{Z}$ is homotopy equivalent to the shifted by -1 Whitney complex (e.g., see [13], p.142). The homology ring of the DGA in this case is the Orlik-Solomon algebra of $L$ after the shift.

2. If $L$ is the intersection lattice of a complex subspace arrangement extrinsically graded by the codimensions of its elements then the DGA $\Delta$ is the DGA from [16] whose homology ring is isomorphic with properly regraded cohomology ring of the subspace complement.

3. Suppose $L=D$ that is the least common multiple lattice from above corresponding to a set $\mathcal{A}$ of homogeneous polynomials. As in the previous sections denote the set of atoms of $D$ by $E$ and put $r=|E|$. Fix a linear order on $E$. Then one defines the extrinsic grading rk $: D \rightarrow \mathbb{N}^{r}$ assigning to each $Q \in D$ the vector of multiplicities of the irreducible factors of $Q$. The complex $\Delta(D)$ is homotopy equivalent to the complex $\oplus_{Q \in D} D_{<Q}$ (cf. [17]).

One can generalize this definition using more general ordered semigroups instead of $\mathbb{N}^{s}$ but we will not use this in the paper.

To show that the example 3 above can be used for computation of the algebra structure on $\operatorname{Tor}_{*}^{S}(S / I, k)$ one can use the well-known graded algebra structure 
on the Taylor complex (see for example [1], p. 6). To define a (bilinear over $S$ ) product on $\tilde{K}$ it is enough to define it on the standard generators. For $\sigma, \tau \subset \mathcal{A}$ we put $\sigma \cdot \tau=0$ if $\sigma \cap \tau \neq \emptyset$ and otherwise

$$
\sigma \cdot \tau=(-1)^{\epsilon(\sigma, \tau)}\left[Q_{\sigma}, Q_{\tau}\right] \sigma \cup \tau
$$

where $[$,$] is the greatest common divisor.$

Theorem 3.2. There is a canonical k-algebra isomorphism $\operatorname{Tor}_{*}^{S}(S / I, k) \rightarrow$ $H_{*}(\Delta(D))$ where the algebra structure in the right hand side is induced by the $D G A$ structure on $\Delta(D)$ defined in Example 3 above.

Proof. It is obvious from definitions that $K(0)$ can be identified with the relative atomic complex $\Delta(D)$. Since any DGA structure on a free resolution can be used for defining the multiplicative structure on Tor, we can use the DGA defined above on $\tilde{K}$. It is straightforward to check that it induces on $\Delta(D)$ the DGA structure defined in Example 3 above.

\section{Minimal resolution}

In this section we fix a set $\mathcal{A}$ of homogeneous polynomials generating ideal $I$ satisfying the condition of Theorem 2.4 and starting from the Taylor resolution $\tilde{K}$ of $S / I$ construct its minimal resolution $\tilde{M}$. The complex $\tilde{M}$ will be realized as a subcomplex of $\tilde{K}$. Moreover we first find a needed subcomplex $M$ of $K$ and then pass to $\tilde{M}$ in the same way as we obtained $\tilde{K}$ from $K$.

Recall that the acyclic complex $K$ has the standard basis consisting of the elements $\sigma$ of the Boolean lattice $\mathcal{B}$ (i.e., subsets of $\mathcal{A}$ ) graded by $|\sigma|$. The monotone map $\phi: \mathcal{B} \rightarrow D$ defines the partition of $\mathcal{B}$ into sets $\mathcal{B}(Q)=\phi^{-1}(Q)$ $(Q \in D)$. Denote by $K(Q)$ the graded subspace of $K$ generated by $\mathcal{B}(Q)$ and notice that $K=\oplus_{Q \in D} K(Q)$ as a graded linear space. If we provide $K(Q)$ with the restriction $d(Q)$ of $d$ it becomes a chain complex. More precisely $d(Q)(a)=$ $\pi_{Q} d(a)$ for every $a \in K(Q)$ where $\pi_{Q}: K \rightarrow K(Q)$ is the canonical projection.

Now we need to make a noncanonical choice. Let $Z(Q)_{p}$ and $B(Q)_{p}$ be the spaces of cycles and boundaries respectively of degree $p$ in $K(Q)$. For each $p$ there are two exact sequences

$$
0 \rightarrow Z(Q)_{p} \rightarrow K(Q)_{p} \rightarrow B(Q)_{p-1} \rightarrow 0,
$$

and

$$
0 \rightarrow B(Q)_{p} \rightarrow Z(Q)_{p} \rightarrow H(Q)_{p} \rightarrow 0 .
$$

We fix a splitting of each of the sequences. In other words we represent

$$
K(Q)_{p}=B(Q)_{p} \oplus H^{\prime}(Q)_{p} \oplus B^{\prime}(Q)_{p-1},
$$

where $B(Q)_{p} \oplus H^{\prime}(Q)_{p}=Z(Q)_{p}$ with the restriction of $d(Q)$ giving an isomorphism $B^{\prime}(Q)_{p-1} \rightarrow B(Q)_{p-1}$ and the restriction of the projection $Z(Q)_{p} \rightarrow$ $H(Q)_{p}$ giving an isomorphism $H^{\prime}(Q)_{p} \rightarrow H(Q)_{p}$.

In the case where the graded subspace $H^{\prime}=\oplus_{Q} H^{\prime}(Q)$ of $K$ is invariant under $d$ we can take this subspace for $M$. However it is easy to find examples where 
this is false (cf. Example 4.2). Our goal is to find a subcomplex $M$ of $K$ that is the graph of a degree -1 linear map $f: H^{\prime} \rightarrow B^{\prime}=\oplus_{Q} B^{\prime}(Q)$. The map $f$ is defined by the following lemma.

Lemma 4.1. For each $a \in H_{p}^{\prime}$ there exists and unique an element $f(a) \in$ $\oplus_{P} B^{\prime}(P)_{p-1}$ such that $d(a+f(a)) \in H^{\prime} \oplus B^{\prime}$.

Proof. Let us prove the uniqueness first. By subtraction we reduce the problem to proving that there is no nonzero $b \in B_{p-1}^{\prime}$ with $d(b) \in H^{\prime} \oplus B^{\prime}$. Suppose such an element $b$ exists and let $P$ be a maximal element in $D$ with the property $b_{P}=$ $\pi_{P}(b) \neq 0$. By the maximality of $P$ we have $d(P)\left(b_{P}\right)=\pi_{P} d(b) \in H^{\prime}(P) \oplus B^{\prime}(P)$ whence $d(P)\left(b_{P}\right)=0$. Since $b_{P} \in B^{\prime}(P)$ we conclude that $b_{P}=0$ which is a contradiction.

Now we prove the existence. Using downward induction on $D$, it suffices to prove the following; let $c \in K_{p}$ be such that $\pi_{P} d(c) \in H^{\prime}(P) \oplus B^{\prime}(P)$ for all $P$ greater than a given $R \in D$. Then there exists $b \in B^{\prime}(R)_{p-1}$ such that $\pi_{P} d(c+b) \in H^{\prime}(P) \oplus B^{\prime}(P)$ for $P \geq R$.

This claim is immediate. Indeed one can take $b \in B^{\prime}(R)_{p-1}$ with the condition that $d(R)(b)=-\left[\pi_{R} d(c)\right]_{B(R)}$ where []$_{B(R)}$ means the projection of $K(R)$ to $B(R)$.

Lemma 4.1 defines a degree -1 linear (by uniqueness) map $f: H^{\prime} \rightarrow B^{\prime}$. Notice that by construction if $a \in H^{\prime}(Q)$ then $f(a) \in \oplus_{P<Q} B^{\prime}(P)$. We put $M=\left\{a+f(a) \mid a \in H^{\prime}\right\}$. Clearly $M$ is a graded linear subspace of $K$.

Lemma 4.2. The subspace $M$ is a subcomplex of $K$.

Proof. We need to prove that $d(M) \subset M$. Suppose $a \in H^{\prime}$ and consider $c=$ $d(a+f(a))$. By construction $c \in H^{\prime} \oplus B^{\prime}$, i.e., $c=e+b$ where $e \in H^{\prime}$ and $b \in B^{\prime}$. Since $d(c)=0 \in H^{\prime} \oplus B^{\prime}$ we have by the uniqueness part of Lemma 4.1 that $b=f(e)$, i.e., $c \in M$.

Now we define the graded free $S$-submodule $\tilde{M}$ of $\tilde{K}$ as generated by $M$. Lemma 4.2 implies that $\tilde{M}$ is a subcomplex of $\tilde{K}$. The following result is the main one of this section.

Theorem 4.3. The complex $\tilde{M}$ is a minimal resolution of $S / I$.

Proof. To prove this theorem it suffices to prove that the complex $\tilde{K} / \tilde{M}$ is exact. For that, in turn, it suffices to prove that $K^{\prime}=(\tilde{K} / \tilde{M}) \otimes k=(\tilde{K} \otimes k) /(\tilde{M} \otimes k)$ is exact.

To analyze the complex $K^{\prime}$ notice first that as graded linear spaces $\tilde{K} \otimes k=K$ and $\tilde{M} \otimes k=M$. Moreover in the decomposition $K=B \oplus H^{\prime} \oplus B^{\prime}$ we have $M \subset H^{\prime} \oplus B^{\prime}$ where $M$ is the graph of $f: H^{\prime} \rightarrow B^{\prime}$. Thus up to natural isomorphism $K^{\prime}=B \oplus B^{\prime}$ as graded linear space. In particular

$$
K^{\prime}=\oplus_{Q}\left(B(Q) \oplus B^{\prime}(Q)\right),
$$

(again as graded linear spaces). Moreover, unlike for $K$, the complexes $K(Q)$ are subcomplexes of $\tilde{K} \otimes k$ whence 4.1 holds in the category of chain complexes, 
i.e., the differential in $K^{\prime}$ coincides with $\oplus_{Q} d(Q)$. Now the statement follows immediately from the isomorphisms $d(Q): B^{\prime}(Q)_{p} \rightarrow B(Q)_{p-1}$.

Since in general the minimal resolution $\tilde{M}$ of $S / I$ is not constructed canonically, it is interesting to consider a case when it is canonical. This resolution was discovered in [4] for so called generic monomial ideals (see below). We want to show how $\tilde{M}$ reduces to this resolution for a significantly wider class of $\mathcal{A}$ (even among monomial ideals).

In [4], the Scarf complex is the subcomplex of $\tilde{M}$ generated by $\sigma \subset \mathcal{A}$ such that $|\mathcal{B}(Q)|=1$ for $Q=Q_{\sigma}$.

Proposition 4.4. The complex $\tilde{M}$ coincides with the Scarf complex (in particular the latter is acyclic) if and only if for every $Q \in D$ either $|\mathcal{B}(Q)|=1$ or the complex $K(Q)$ is exact.

Proof. The condition is obviously necessary. Let us prove that it is sufficient. Put $\bar{D}=\{Q \in D|| \mathcal{B}(Q) \mid=1\}$. We have $K(Q)=K_{1}(Q)=H_{1}^{\prime}(Q)$ for every $Q \in \bar{D}$ and $H^{\prime}(Q)=0$ for other $Q$. Thus the following claim suffices for the proposition.

Claim. Let $\sigma \subset \mathcal{A}$ be such that $Q_{\sigma} \in \bar{D}$. Then $Q_{\sigma_{i}} \in \bar{D}$ for every $Q_{i} \in \sigma$.

Proof of Claim. Suppose $Q_{\sigma_{i}} \notin \bar{D}$ for some $i$. Then there exists $Q_{j} \in \mathcal{A}$ such that $Q_{j}$ divides $Q_{\sigma_{i}}$. There are two possibilities. One possibility is that $Q_{j} \in \sigma_{i}$. This implies $Q_{\sigma_{j}}=Q_{\sigma}$ which is a contradiction. The other possibility is that $Q_{j} \notin \sigma_{i}$. If $Q_{j} \in \sigma$ then $j=i$ and $Q_{\sigma}=Q_{\sigma_{i}}$ which is a contradiction. If $Q_{j} \notin \sigma$ then $Q_{\sigma}=Q_{\sigma \cup\left\{Q_{j}\right\}}$ which is again a contradiction. This proves the claim and the proposition.

In [4], a monomial ideal $I$ is called generic if no variable appears with the same nonzero exponent in two distinct minimal generators of $I$. This (though ambiguous) term can be used for an arbitrary set $\mathcal{A}$ of homogeneous polynomials (with respect ot their factorizations into irreducible factors).

Proposition 4.5. Let $\mathcal{A}$ be generic in the above sense. Then for every $Q \in D$ the poset $\mathcal{B}(Q)$ is Boolean.

Proof. The key observation is that $\mathcal{B}(Q)$ has a unique minimal element. Indeed write $Q=\prod_{i=1}^{r} Z_{i}^{m_{i}}$ where $Z_{i}$ are irreducibles and $m_{i}>0$. Then for each $i$ there exists a unique $Q_{j_{i}} \in \mathcal{A}$ such that $Z_{i}$ has exponent $m_{i}$ in decomposition of $Q_{j_{i}}$. Put $\sigma=\left\{Q_{j_{i}} \mid i=1, \ldots, r\right\}$. It is easy to see that $Q_{\sigma}=Q$ and $\sigma$ is the unique minimal element of $\mathcal{B}(Q)$.

Now the result follows since $\mathcal{B}(Q)$ always has a unique maximal element and with any two elements of $\mathcal{B}$ contains every element between them.

Corollary 4.6. If $\mathcal{A}$ is generic then $\tilde{M}$ coincides with the Scarf complex (cf. [4]). 
It is easy to find examples with nongeneric $\mathcal{A}$ and all Boolean $\mathcal{B}(Q)$ (e.g., $\mathcal{A}=\{x y, x z\})$. Moreover Propositions 4.4 and 4.5 give a class of $\mathcal{A}$ with the Scarf resolution (significantly wider than generic) defined by an easily checkable condition. The condition is:

$$
Q_{\sigma}=Q_{\tau} \text { implies } Q_{\sigma \cap \tau}=Q_{\sigma} \text { for every subsets } \sigma \text { and } \tau \text { of } \mathcal{A} \text {. }
$$

The following example shows that $\mathcal{B}(Q)$ do not have to be Boolean to satisfy the condition of Proposition 4.4.

Example 4.7. Let $\mathcal{A}=\left\{x^{2} y z, x y^{2} w, x^{2} z w, x y^{2} z\right\}$. One can find easily that the condition of Proposition 4.4 holds whence the minimal resolution coincides with the Scarf complex. On the other hand $\mathcal{B}(Q)$ for $Q=x^{2} y^{2} z w$ has three minimal elements.

Now we give a couple of examples with $H^{\prime}$ not being invariant with respect to the differential (in particular nonvanishing).

Example 4.8. Let $\mathcal{A}=\{x y, x z, y u, u v\}$ enumerated in the order they are written. There are three $\mathcal{B}(Q)$ that have more than one element: $\mathcal{B}(x y z u)=$ $\{\{2,3\},\{1,2,3\}\}, \mathcal{B}(x y u v)=\{\{3,4\},\{1,3,4\}\}$, and

$\mathcal{B}($ xyzuv $)=\{\{1,2,4\},\{2,3,4\},\{1,2,3,4\}\}$. Only the last $K(Q)$ has nontrivial homology, namely $\operatorname{dim} H_{3}=1$ and as a cycle representative of a nonzero class one can take $\sigma=\{1,2,4\}$. Then one has $d(\sigma)=\{1,2\}-\{1,4\}+\{2,4\}$ where $\{1,4\}$ is the trivial cycle in $K(x y u v)$, namely the boundary of $\tau=$ $-\{1,3,4\}$. Thus using our construction we have $M_{3}=k a$ where $a=\sigma+\tau$ and then $d(a)=\{1,2\}+\{2,4\}-\{1,3\}-\{3,4\} \in M_{2}$ where $M_{2}$ is generated by $\{\{1,2\},\{1,3\},\{2,4\},\{3,4\}\}$. In particular the Betti numbers of $S / I$ are $1,4,4,1$.

Example 4.9. This is Avramov's example from [1]. Let

$$
\mathcal{A}=\left\{x^{2}, x y, y z, z w, w^{2}\right\}
$$

again with the natural linear order. There are $\operatorname{six} \mathcal{B}(Q)$ with more than one element:

$$
\begin{aligned}
\mathcal{B}\left(x^{2} y z\right) & =\{\{1,3\},\{1,2,3\}\} \\
\mathcal{B}(x y z w) & =\{\{2,4\},\{2,3,4\}\}, \\
\mathcal{B}\left(y z w^{2}\right) & =\{\{3,5\},\{3,4,5\}\}, \\
\mathcal{B}\left(x^{2} y z w\right) & =\{\{1,2,4\},\{1,3,4\},\{1,2,3,4\}\}, \\
\mathcal{B}\left(x y z w^{2}\right) & =\{\{2,3,5\},\{2,4,5\},\{2,3,4,5\}\},
\end{aligned}
$$

and

$$
\mathcal{B}\left(x^{2} y z w^{2}\right)=\{\{1,3,5\},\{1,2,3,5\},\{1,2,4,5\},\{1,3,4,5\},\{1,2,3,4,5\}\} .
$$

Only the last three give $K(Q)$ with nonvanishing homology. As cycle representatives one can take $\{1,2,4\},\{2,4,5\},\{1,2,4,5\}$. Then one gets the generators of $M_{4}$ as $\{1,2,4,5\}$, of $M_{3}$ as $\{1,2,4\}+\{2,3,4\},\{2,4,5\}+\{2,3,4\},\{1,2,5\},\{1,4,5\}$ and of $M_{2}$ as all the pairs of generators except $\{1,3\},\{2,4\}$, and $\{3,5\}\left(M_{1}=K_{1}\right.$ and $M_{0}=K_{0}$ as always). It is easy to see that $M$ is invariant under $d$ and the 
dimensions of $M_{p}$ coincide with the respective Betti numbers of $S / I$ that are $1,5,7,4,1$.

\section{Examples of ideals with Taylor resolution}

In this section we show examples of classes of sets $\mathcal{A}$ of homogeneous polynomials such that the conditions of Theorem 2.4 hold whence the respective Taylor complex is exact. The following result is useful for that. For every $P \in W$ denote by $E_{P}$ the subset of $E$ of the irreducible polynomials taking part in the factorization of $P$. Also put $J(P)=J\left(E_{P}\right)$.

Proposition 5.1. For every saturated $G \subset E$ and every $P \in W(G)$ we have $\tilde{H}_{p}(D(G,<P))=0$ for $p \geq\left|E_{P}\right|-1$.

Proof. Suppose $G$ and $P$ are as in the statement and $P=\prod_{i=1}^{r} Z_{i}^{m_{i}}$ is the factorization of $P$ into irreducible factors. Notice that $r=\left|E_{P}\right|$. Let now $X$ and $Y$ be distinct maximal elements of $D(G, P)$. Then $\psi_{G}(X \vee Y) \geq P$. This implies that for every $i, 1 \leq i \leq r$, all but at most one maximal elements of $D(G, P)$ have the factor $Z_{i}^{\ell_{i}}$ with $\ell_{i}<m_{i}$ in their factorizations. Since on the other hand each element of $D(G, P)$ has the multiplicity of at least one of $Z_{i}$ smaller than $m_{i}$ we have

$$
|\max D(G, P)| \leq r
$$

Now the poset $D(G, P)$ can be viewed as a lattice with the maximal and minimal elements deleted. Thus by [9] its homology can be computed as the homology of the coatomic complex of the lattice. The vertices of this complex are the maximal elements of $D(G, P)$ whence (5.1) implies that either this complex is a simplex or its dimension is less than $r-1$. The result follows.

Corollary 5.2. If the set $E_{P}$ forms a regular sequence (for the module $S$ ) then the condition of Theorem 2.4 holds for $P$ and any $G$ such that $P \in \psi_{G}(D)$.

Proof. We have in this case $\operatorname{depth} J(G) \geq \operatorname{depth} J(P)=\left|E_{P}\right|$. Thus the result follows from the previous proposition.

In particular Corollary 5.2 recovers Taylor's result that for any monomial ideal the Taylor complex is acyclic.

Another class of ideals where the condition of Theorem 2.4 simplifies (although stays nontrivial) consists of ideals generated by products of linear polynomials. For this class the condition of Theorem 2.4 specializes to

$$
\tilde{H}_{p}(D(G, P))=0 \text { for } p \geq \operatorname{dim} E_{P}-1,
$$

where $\operatorname{dim} E_{p}$ is the usual dimension of the linear space generated by $E_{P}$.

We can find a sufficient condition for (5.2) using a result similar to (although more subtle than) Proposition 5.1 but for atomic complexes. First we need a lemma. 
Lemma 5.3. Let $G$ be a saturated subset of $E$ and $U$ a decreasing subset of the subposet $\psi_{G}(D)$ of $W(G)$. Then $\psi_{G}$ is a homotopy equivalence of $\psi_{G}^{-1}(U)$ and $U$.

Proof. As in the proof of Lemma 2.2 it is easy to see that for every $P \in U$ the poset $\psi_{G}^{-1}\left(U_{\leq P}\right)$ has the unique maximal element $\operatorname{lcm}\left\{Q_{i} \in \mathcal{A} \mid \psi_{G}\left(Q_{i}\right) \leq P\right\}$. Thus this poset is contractable and the result follows.

For each $i=1,2, \ldots, m$ put $E_{i}=E_{Q_{i}}$.

Proposition 5.4. Suppose all $Q_{i}$ are square free products of linear polynomials. If for every $i=1,2, \ldots, m$ the set $G_{i}=E \backslash E_{i}$ is saturated (that in this case means no element of $E_{i}$ is a linear combination of elements from $G_{i}$ ) then (5.2) holds.

Proof. Fix a saturated set $G \subset E$ and $P \in W(G)$. Lemma 5.3 implies that $\tilde{H}_{p}(D(G, P)) \approx \tilde{H}_{p}\left(\psi_{G}(D)_{<P}\right)$ for every $p$. Let $s$ be the number of the minimal elements $Z_{i_{1}}, \ldots, Z_{i_{s}}$ of $\psi_{G}(D)_{<P}$, i.e., $Z_{i_{j}}=\psi_{G}\left(Q_{i_{j}}\right)$ and $Z_{i_{j}}<P$. If $s \leq$ $\operatorname{dim} E_{P}$ then the dimension of the atomic complex of $\psi_{G}(D)_{<P}$ is less than $\operatorname{dim} E_{P}-1$ unless this complex is a simplex (cf. the proof of Proposition 5.1). Then (5.2) follows.

Thus we have to prove only the impossibility of $s>\operatorname{dim} E_{P}$. Suppose it is the case. Let $r$ be the largest dimension of $G_{i}$. Then the conditions on $G_{i}$ and $Q_{i}$ imply that $\operatorname{dim} \bigcap_{j=1}^{s} G_{i_{j}} \leq r-s+1<r-\operatorname{dim} E_{P}+1$. The condition $Z_{i_{j}}<P$ is equivalent to $G_{i_{j}} \supset E \backslash E_{P}$ whence $\bigcap_{j=1}^{s} G_{i_{j}} \supset E \backslash E_{P}$. We obtain

$$
\operatorname{dim}\left(E \backslash E_{P}\right)<r-\operatorname{dim} E_{P}+1,
$$

whence

$$
\operatorname{dim} E \leq \operatorname{dim} E_{P}+\operatorname{dim}\left(E \backslash E_{P}\right)<r+1
$$

which is a contradiction.

The condition (5.2) simplifies significantly if we assume that the set $E$ of linear polynomials is generic, i.e., any subset of $E$ with at most $n$ elements is linearly independent.

Proposition 5.5. Suppose that all $Q_{i}$ are products of linear polynomials and $E$ is generic. Then (5.2) is equivalent to

$$
\oplus_{P \in D} \tilde{H}_{p}\left(D_{<P}\right)=0 \text { for } p \geq n-1 .
$$

Equality (5.3) for $P \in D$ with $\mathrm{rk} E_{P}=n$ suffices for (5.2).

Proof. Suppose first that $G$ is a proper saturated subset of $E$ and $P \in W(G)$. Since $E$ is generic, $G$ is linearly independent and so is $E_{P} \subset G$, i.e., rk $E_{P}=\left|E_{P}\right|$. Proposition 5.1 implies (5.2) for this $G$ and $P$.

Now suppose that $G=E$ whence $\psi_{G}$ is the canonical embedding $D \subset W$. Then the only nontrivial case is where $P \in D$ whence $D(G,<P)=D_{<P}$. If $\mathrm{rk} E_{P}<n$ then $E_{P}$ is again linearly independent and (5.2) holds by the 
same reason as in the previous paragraph. If $\operatorname{rk} E_{P}=n$ then (5.2) is obviously equivalent to (5.3).

Notice that equality (5.3) uses only the combinatorics of the lattice $D$ and $n$. This combinatorics can be expressed in terms of other polynomial ideals, the simplest from them being monomial ones. More precisely assign to each linear polynomial $Z_{i} \in E(i=1,2, \ldots, r)$ an indeterminant $y_{i}$ and consider the polynomial $\operatorname{ring} \tilde{S}=k\left[y_{1}, \ldots, y_{r}\right]$. The natural algebra map $\tilde{S} \rightarrow S$ via $y_{i} \mapsto Z_{i}$ assigns to each $Q_{j} \in \mathcal{A}$ a monomial $\tilde{Q}_{j} \in \tilde{S}$ which generate the monomial ideal $\tilde{I}$ of $\tilde{S}$. Then the following result follows straightforwardly from the results of the previous sections.

Corollary 5.6. Under the conditions of Proposition 5.5 the Taylor complex of $S / I$ is a resolution if and only if $b_{p}(\tilde{S} / \tilde{I})=0$ for $p \geq n+1$. If this condition holds then the $k$-algebras $\operatorname{Tor}_{*}^{S}(S / I, k)$ and $\operatorname{Tor}_{*}^{\tilde{S}}(\tilde{S} / \tilde{I}, k)$ are naturally isomorphic.

Using Proposition 5.5, we can easily give a series of examples of nonmonomial ideals whose Taylor complexes are exact.

Example. Consider generic arrangement of more than $n$ hyperplanes in a space of dimension $n$. Let $\mathcal{A}$ consist of no more than $n$ arbitrary products of functionals of hyperplanes. Then the Taylor complex of $\mathcal{A}$ is exact. Indeed for every $P \in D_{0}$ the poset $D_{<P}$ has at most $n$ atoms whence its atomic complex is either a simplex or has dimension less than $n-1$. The condition (5.3) follows.

Remark 5.7. In the case where $Q_{i}$ are square free products of linear polynomials the atomic complex of $D_{<P}$ can be interpreted as the nerve of the collection $\left\{G_{i}=E_{P} \backslash E_{i} \mid E_{i} \subset E_{P}\right\}$ of subsets of $E_{P}$. This is the reason for the relations between the Betti numbers of $S / I$ and the complement of a coordinate subspace arrangement studied for monomial ideals in [10]. In particular the duality between $E_{i}$ and $G_{i}$ leads to the appearance of the Alexander dual complexes.

\section{References}

[1] L. Avramov, Obstructions to the existence of multiplicative structures on minimal free resolutions, Amer. J. Math. 103 (1981), 1-32.

[2] E. Babson and C. Chan, personal communication.

[3] K. Baclawski, Whitney numbers of geometric lattices, Advances in Math. 16 (1975), 125138.

[4] D. Bayer, I. Peeva, and B. Sturmfels, Monomial resolutions, Math. Res. Lett. 5 (1998), $31-46$.

[5] A. Björner, Topological methods, in Handbook of combinatorics (R. Graham et al eds.), pp. 1819-1872, North-Holland Press, Amsterdam, 1994.

[6] M. De Longueville, The ring structure on the cohomology of coordinate subspace arrangements, preprint, 1998.

[7] D. Eisenbud, Commutative algebra with a view toward algebraic geometry, SpringerVerlag, New York-Berlin, 1995.

[8] E.M. Feichtner, Cohomology algebras of subspace arrangements and of classical configuration spaces, Cuvillier Verlag Göttingen, 1997 (Doctors Dissertation at TU, Berlin).

[9] J. Folkman, The homology groups of a lattice, J. Math. Mech. 15 (1966), 631-636. 
[10] V. Gasharov, I. Peeva, V. Welker, Coordinate subspace arrangements and monomial ideals, preprint, 1998.

[11] _ The lcm-lattice in monomial resolutions, preprint, 1998.

[12] D.G. Northcott, Finite free resolutions, Cambridge Tracts in Mathematics, No. 71, Cambridge University Press, Cambridge, 1976.

[13] P. Orlik and H. Terao, Arrangements of hyperplanes, Springer-Verlag, Berlin, 1992.

[14] D. Quillen, Homotopy properties of the poset of nontrivial p-subgroups of a group, Advances in Math. 28 (1978), 101-128.

[15] S. Yuzvinsky, On generators of the module of logarithmic 1-forms with poles along an arrangement, J. Algebraic Combin. 4 (1995), 253-269.

[16] _ Small rational model of subspace complement, preprint, math.C0/9806143.

[17] _ Rational model of subspace complement on atomic complex, to appear in Belgrade Journal of Math.

University of Oregon, Eugene, OR 97403

E-mail address: yuz@math.uoregon.edu 